\title{
Philosophiques
}

\section{Index volume XXIII — 1996}

Volume 24, numéro 1, printemps 1997

Avez-vous lu Rawls?

URI : https://id.erudit.org/iderudit/027446ar

DOI : https://doi.org/10.7202/027446ar

Aller au sommaire du numéro

Éditeur(s)

Société de philosophie du Québec

ISSN

0316-2923 (imprimé)

1492-1391 (numérique)

Découvrir la revue

Citer ce document

(1997). Index volume XXIII - 1996. Philosophiques, 24(1), 225-227.

https://doi.org/10.7202/027446ar

Ce document est protégé par la loi sur le droit d'auteur. L'utilisation des services d'Érudit (y compris la reproduction) est assujettie à sa politique d'utilisation que vous pouvez consulter en ligne.

https://apropos.erudit.org/fr/usagers/politique-dutilisation/
Cet article est diffusé et préservé par Érudit.

Érudit est un consortium interuniversitaire sans but lucratif composé de l’Université de Montréal, l'Université Laval et l'Université du Québec à Montréal. Il a pour mission la promotion et la valorisation de la recherche. https://www.erudit.org/fr/ 


\section{INDEX \\ VOLUME XXIII - 1996}

Numéro 1 (Printemps) :

Numéro 2 (Automne) :

\section{ARTICLES}

Éric Bourneuf, Pour une gnoséologie objective et applicable ........ 265

Daniel Charles, Pour une esthétique sans privilèges

Daniel Dumouchel, La dialectique du beau et du sublime:

l'héritage kantien d'Adorno.

Suzanne Foisy, Présentation du numéro thématique

Critères esthétiques et métamorphoses du beau

Suzanne Foisy, Différence esthétique et argumentation

Jean-Luc Gouin, La Raison comme lemme philosophique

ou " Der Instinkt der Vernünftigkeit "-Pour accoster Hegel .. 285

Peter Hadreas, Searle versus Derrida?

Mette Hjort, Le privilège culturel

et la politique de la reconnaissance.

David Guy Joannis, Le projet épistémologique de Sartre 305

Danielle Lories, Art contemporain:

questions nouvelles pour l'esthétique ?............................. 15

Peter McCormick, Le sublime et la fiction.................................. 93

Christoph Menke, Le regard esthétique.

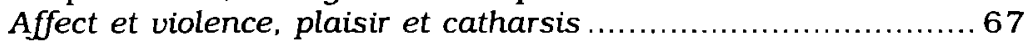

Roberto Miguelez, Kant et la nature ....................................... 253

Herman Parret, Le plaisir esthétique et la vérité des sens.............81

Pablo da Silveira, Deux conceptions de la neutralité de l'État..... 227

Jean-Jacques Wunenburger, Transfiguration et défiguration

du corps souffrant. Les métamorphoses de l'idéal

de beauté physique dans les arts plastiques.

BIBLIOGRAPHIE sèlective sur la question des critères esthétiques. 133

\section{ILLUSTRATIONS}

Francis Bacon, Tryptique d'aoūt 1972 (hors-texte) ........ XXIII n ${ }^{\circ} 1$

G. Schloesser, Correspondances interloquées, 1994.............. 13

J. Cirincione, B. D'Amato et M. Ferraro,

The Imperial Message : Grid with Characters, 1993-1994_..... 14

J. Jones, Malerei von Ort.............................................. 131

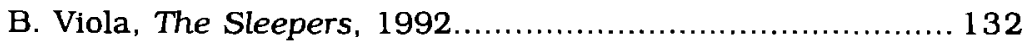




\section{INTERVENTIONS}

Josiane Boulad-Ayoub, De David et Jean-Jacques à Jean et à Jacques. Un échange inconnu entre Hume et Rousseau.. 151

Marcel Côté et Gilles Paradis, Les dictionnaires généraux de philosophie en langue française...

Jean-Paul Forster, Avec les yeux d'un étranger :

Les Lettres d'un Persan de George Lyttleton.

Christian Godin, L'encyclopédisme hors d'Europe

Dominique Janicaud, Le temps de la nature et la mesure du temps.

\section{TABLES RONDES}

Descartes : le quatrième centenaire

Sous la direction de François Duchesneau.

Daniel Dumouchel, Descartes : discours et méthode.

François Duchesneau, Le modèle du vivant dans la physique cartésienne.

Josiane Boulad-Ayoub, Les récurrences du platonisme chez Descartes.

Georges J. D. Moyal, L'hypothèse du rêve et les vérités de la raison.

\section{ÉTUDES CRITIQUES}

Mario Dufour, Claude Lévesque, Le proche et le lointain. Essais.

Jean-Marc Narbonne, Stanley Rosen, The Question of Being.

A Reversal of Heidegger.

\section{COMPTES RENDUS}

Jacques Bidet, Théorie de la modernité.

Suivi de : Marx et le marché (Gilles Labelle)..................... 449

Anne Cauquelin, La mort des philosophes et autres contes

(Fernand-Luc Bergeron)

Gilles Deleuze et Fëlix Guattari, Qu'est-ce que la philosophie?

(Mario Dufour).

Pascal Engel, Lire Davidson. Interprétation et holisme

(Richard Vallée).

Manfred Frank, Jean-Paul Larthomas \& Alexis Philonenko,

Sur la Troisième Critique (Daniel Dumouchel)

Jean Greisch, Ontologie et temporalité. Esquisse d'une interprétation intégrale de Sein und Zeit (Yvon Corbeil)

Jean Grondin, L'horizon herméneutique de la pensée contemporaine (Marc Imbeault) 
Otfried Hōffe, Principes du droit

(Daniel M. Weinstock)

Raymond Klibanski et David Pears, La philosophie en Europe

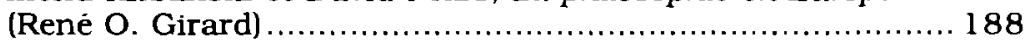

Angèle Kremer-Marietti, La philosophie cognitive

(Jean-Pierre Marquis).

Josette Lanteigne, La question dujugement?

(Candida Jaci de Sousa Melo).

Christian Lazzeri, Force et justice dans la politique de Pascal

(Christian Nadeau).

Béatrice Longuenesse, Kant et le pouvoir de juger. Sensibilité et discursivité dans l'Analytique transcendantale de la Critique de la raison pure (Claude Piché)....

Alasdair MacIntyre, Quelle justice? Quelle rationalité ? (André Lacroix).

Philip M. Merklinger, Philosophy, Theology and Hegel's Berlin Philosophy of Religion (Mylène Dufour).............................. 446

Carlo Michelstaedter, La persuasion et la rhétorique (Steve Light).

Gérard Naddaf, L'origine et l'évolution du concept grec de phusis (Germain Derome)

Thomas Nagel, Égalité et partialité

(Paul Dumouchel).

Michel Paty, Einstein philosophe

(Yvon Gauthier).

Jacques Piquemal, Essais et leçons d'histoire de la médecine et de la biologie (Daniel Teysseire)....

Serge Robert, Les mécanismes de la découverte scientifique (Don Ross)

Chantal Saint-Jarre, Du Sida, l'anticipation imaginaire de la mort et sa mise en discours (Isabelle Lasvergnas).

Frederic M. Schroeder, Form and Transformation.

A Study in the Philosophy of Plotinus (Georges Leroux) 